\title{
A comparison of pop and chop to divide and conquer in resident cataract surgery
}

\author{
This article was published in the following Dove Press journal: \\ Clinical Ophthalmology \\ 21 September 2016 \\ Number of times this article has been viewed
}

\section{Fredric J Gross \\ Debra E Garcia-Zalisnak \\ Courtney E Bovee \\ Joy D Strawn}

Department of Ophthalmology, Eastern Virginia Medical School, Norfolk, VA, USA
Correspondence: Fredric J Gross Department of Ophthalmology, Eastern Virginia Medical School, PO Box 1980, Norfolk, VA 2350I-1980, USA

Tel +l 7576202309

Email heye5@aol.com

\begin{abstract}
In this randomized prospective study, the cumulative dissipated energy and case time of pop and chop and of traditional four-quadrant divide and conquer in the first 60 cases (in total 120 eyes) of cataract surgery performed by two residents at the Veterans Administration Hospital in Hampton, Virginia, were compared. Overall and individually, the residents had significantly shorter case times and used significantly less cumulative dissipated energy for performing pop and chop than that for divide and conquer technique. There was no difference in complication rates or visual outcomes between these two techniques. The results of this study suggest that pop and chop is a more time- and energy-efficient method of nucleofractis than divide and conquer for novice resident surgeons.
\end{abstract}

Keywords: case time, cataract surgery, phacoemulsification, cumulative dissipated energy

\section{Introduction}

Teaching residents to perform cataract surgery is one of the most important and challenging tasks in an ophthalmology training program. Since its introduction by Howard Gimbel in 1991, four-quadrant divide and conquer has become the standard method for teaching resident surgeons to safely and effectively remove the nucleus during cataract surgery. ${ }^{1,2}$ To date, only few studies have analyzed other methods of nuclear disassembly for novice surgeons. In this study, the safety and efficiency of teaching pop and chop as an alternative method to divide and conquer to novice resident surgeons were examined.

\section{Methods}

This is a prospective and randomized study of resident cataract surgery using two different techniques of nucleofractis performed at the Veterans Administration Hospital in Hampton, Virginia. This study was approved by the Institutional Review Boards of the Veterans Administration Hospital and Eastern Virginia Medical School. All patients received and signed informed consent prior to their cataract surgery. The first 60 cases performed by two senior residents between July 2014 and December 2015 were randomly assigned to pop and chop or divide and conquer phacoemulsification using a random-number generator. In all the cases, the resident was the primary surgeon and was assisted by three different attending surgeons. Two attending surgeons supervised the divide and conquer cases, whereas a third surgeon assisted with the pop and chop cases. All attending surgeons had $>20$ years of experience in performing and teaching divide and conquer. The attending surgeon who taught pop and chop had used and taught this method for 10 years. The Alcon Centurion System for cataract extraction was used in this technique. Patients with comorbidities such 
Table I Exclusion criteria

\begin{tabular}{llll}
\hline & PC & DC & Total \\
\hline Enrolled patients (n) & 60 & 60 & 120 \\
Excluded patients (total) & 7 & 6 & 13 \\
IFIS & 2 & 2 & 4 \\
Posterior synechiae & 1 & 1 & 2 \\
Previous surgery/trauma & 0 & 0 & 0 \\
Pseudoexfoliation & 0 & 0 & 0 \\
Intraoperative complications & 4 & 3 & 7 \\
\hline
\end{tabular}

Abbreviations: DC, divide and conquer; IFIS, intraoperative floppy iris syndrome; PC, pop and chop.

as intraoperative floppy iris syndrome, posterior synechiae, pseudoexfoliation, and previous eye surgery or trauma were excluded from this study. In addition, patients who experienced surgical complications such as vitreous loss, capsular rupture, or zonular dehiscence were also excluded from the analysis. Patient demographic data, cumulative dissipated energy (CDE), case times, and best corrected postoperative visual acuity were recorded and analyzed for the study cohort.

\section{Results}

Data from 120 cataract surgeries performed by two resident surgeons were examined in this study. In total, 13 cases who met the exclusion criteria were eliminated from the study (Table 1); six patients were excluded for comorbidities, and seven were excluded for intraoperative complications. Of the 107 cases studied, 53 (49.5\%) were operated by Surgeon 1 and $54(50.5 \%)$ by Surgeon 2; 54 cases (50.5\%) were operated by using divide and conquer, whereas the remaining 53 cases $(49.5 \%)$ were operated by using pop and chop. Demographic data such as age, race, and sex were similar in these two groups (Table 2). Average nuclear density was 3.0 in both the groups (Table 3 ).

Figure 1 shows the analysis of CDE data of each surgeon separately and for the total samples included in this study. Surgeon 1 had an average CDE of 15.0 (95\% confidence

Table 2 Patient demographics

\begin{tabular}{llll}
\hline & PC & DC & Total \\
\hline Analysis group $(\mathrm{n})$ & 53 & 54 & 107 \\
$\begin{array}{l}\text { Average age, mean } \pm \mathrm{SD} \text {, years } \\
\text { Sex, } \mathrm{n}(\%)\end{array}$ & $67.3 \pm 7.9$ & $69.1 \pm 10.2$ & $68.2 \pm 9.2$ \\
$\quad \begin{array}{lll}\text { Female } \\
\quad \text { Male }\end{array}$ & $3(5.7)$ & $6(11 . \mathrm{l})$ & $9(8.4)$ \\
Race, $\mathrm{n}(\%)$ & $50(94.3)$ & $48(88.9)$ & $98(91.6)$ \\
$\quad$ Black & & & \\
$\quad \begin{array}{l}\text { Pacific Islander } \\
\text { White }\end{array}$ & $13(24.5)$ & $16(29.6)$ & $29(27.1)$ \\
\hline
\end{tabular}

Abbreviations: DC, divide and conquer; PC, pop and chop; SD, standard deviation.
Table 3 Nuclear density

\begin{tabular}{lll}
\hline $\begin{array}{l}\text { Grade of } \\
\text { nuclear sclerosis }\end{array}$ & DC & PC \\
\hline 0 & 0 & 1 \\
\hline & 5 & 3 \\
2 & 10 & 11 \\
3 & 19 & 20 \\
4 & 20 & 18 \\
Average & 3.0 & 3.0 \\
\hline
\end{tabular}

Abbreviations: DC, divide and conquer; PC, pop and chop.

interval $[\mathrm{CI}]=11.8-19.2)$ for the divide and conquer method and $11.1(95 \% \mathrm{CI}=8.7-14.2)$ for the pop and chop method; this difference was not statistically significant $(P=0.0888)$. Surgeon 2 had an average CDE of 16.7 (95\% $\mathrm{CI}=12.1-23.1)$ for the divide and conquer method and $6.1(95 \% \mathrm{CI}=4.4-8.5)$ for the pop and chop method; this difference was statistically significant $(P<0.0001)$. When the data of both the surgeons were combined together, the average $\mathrm{CDE}$ was 15.9 (95\% $\mathrm{CI}=12.9-19.6)$ for the divide and conquer method and $8.6(95 \% \mathrm{CI}=7.0-10.7)$ for the pop and chop method; this difference was statistically significant $(P<0.001)$.

Figure 2 shows the analysis of case time data of each surgeon separately and for the total samples included in this study. On average, case times for Surgeon 1 were 27.8 minutes (95\% CI $=24.9-31.0)$ for the divide and conquer method and 15.0 minutes ( $95 \% \mathrm{CI}=13.4-16.7)$ for the pop and chop method; this difference was statistically significant $(P<0.0001)$. Case times for Surgeon 2 were 34.4 minutes $(95 \% \mathrm{CI}=30.0-39.4)$ for the divide and conquer method and 20.5 minutes $(95 \%$ $\mathrm{CI}=17.9-23.5)$ for the pop and chop method; this difference was statistically significant $(P<0.0001)$. When the data of

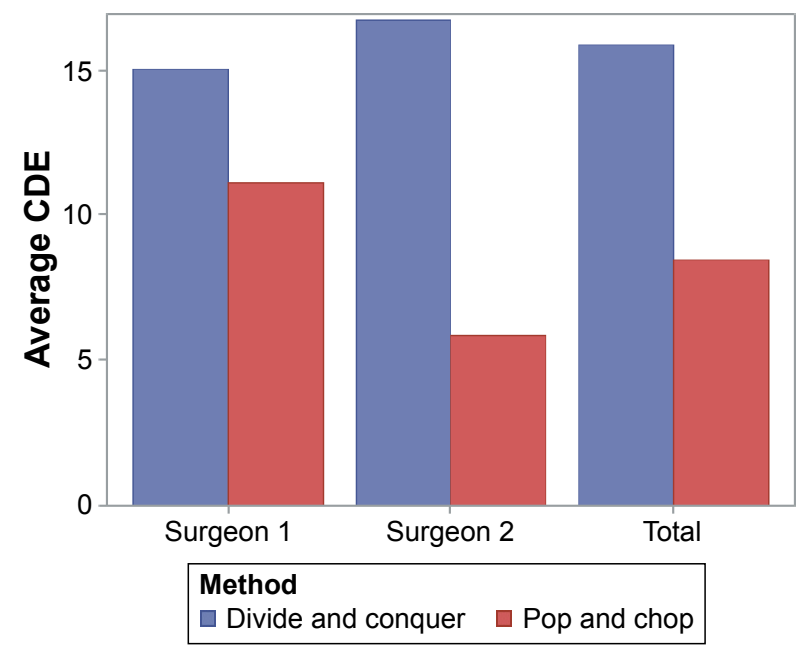

Figure I Average CDE per method for each surgeon and total sample. Abbreviation: $\mathrm{CDE}$, cumulative dissipated energy. 


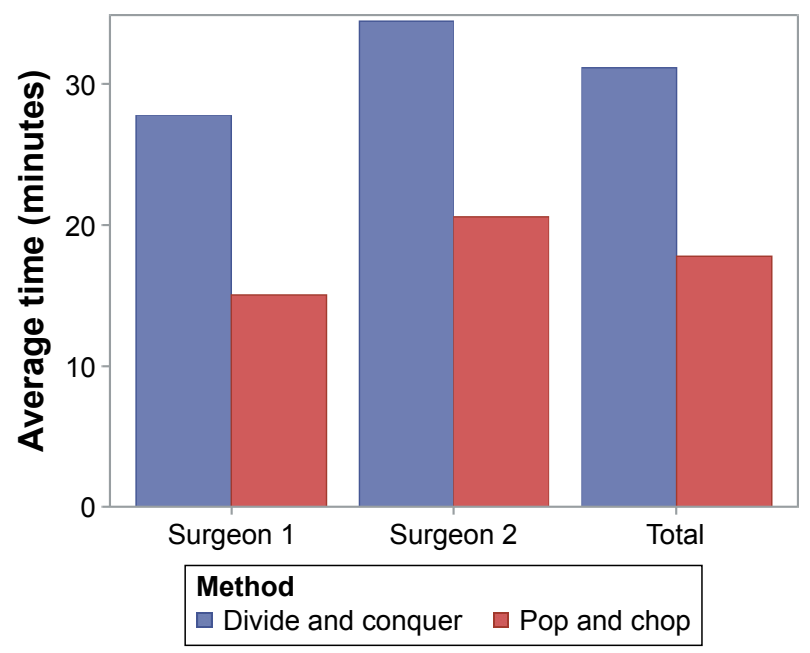

Figure 2 Average time per method for each surgeon and the total sample.

both the surgeons were combined together, the average case time was 31.1 minutes $(95 \% \mathrm{CI}=28.3-34.2)$ for the divide and conquer method and 17.8 minutes ( $95 \% \mathrm{CI}=16.2-19.6)$ for the pop and chop method; this difference was statistically significant $(P<0.0001)$.

Final best corrected visual acuity of $\geq 20 / 40$ was achieved in $90 \%$ of patients in the pop and chop group and $95 \%$ in the divide and conquer group. After excluding patients with preexisting retinal pathologies (five had age-related macular degeneration and one had a macular hole), $100 \%$ of patients in the pop and chop group and $99 \%$ of the patients in the divide and conquer group achieved 20/40 or better vision (Figure 3).

Intraoperative complications occurred in four (7.5\%) pop and chop cases and three (5.6\%) divide and conquer cases. These cases had two posterior capsular tears and two zonular

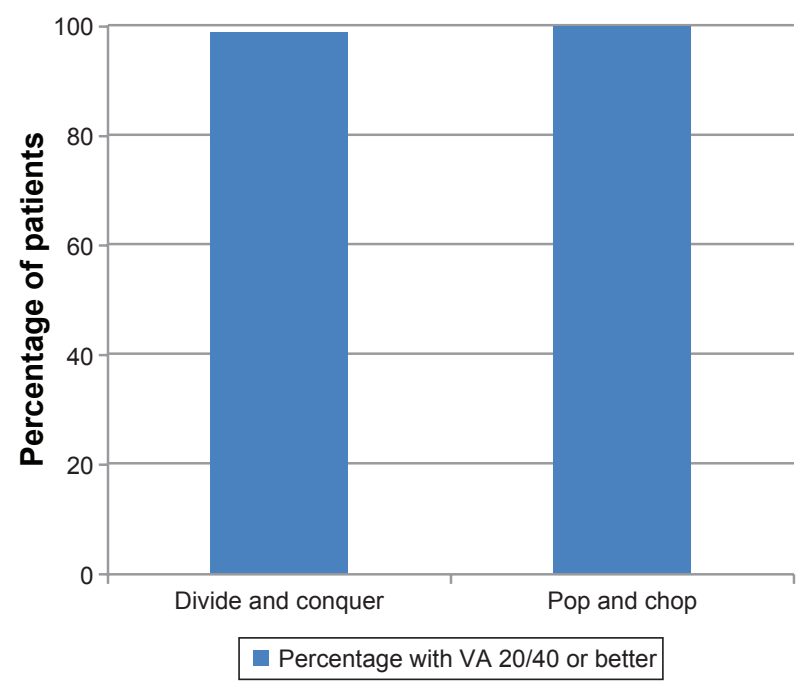

Figure 3 Final best corrected VA. Abbreviation: $V A$, visual acuity. dehiscences with pop and chop method and three posterior capsular tears with divide and conquer method.

\section{Discussion}

Pop and chop is a supracapsular method of nuclear chopping initially described by Pandit and Oetting. ${ }^{3}$ In this technique, nucleus is partially prolapsed out of the capsular bag during hydrodissection and then divided between the phaco tip and a second instrument into heminuclei or quadrants that can easily be removed by phaco-assisted aspiration in the pupillary plane or anterior chamber. Because nuclear fragmentation is performed under direct visualization above the capsular bag, it is easier to learn and perform than endocapsular chopping techniques that require significant surgical experience and skill. ${ }^{4}$ In a retrospective chart review comparing pop and chop with divide and conquer, Parlitsis et al concluded that both the techniques are appropriate for resident surgeons and noted no significant differences in visual outcomes or complication rates. ${ }^{5}$ In the present study, the safety and efficacy of teaching pop and chop to resident surgeons were confirmed. In addition, we report that pop and chop is a significantly more efficient surgical technique than divide and conquer, which requires lesser phaco energy and surgical time than divide and conquer when performed early in residency training.

Supranuclear methods of nucleofractis have used lesser energy than divide and conquer when performed by experienced surgeons. ${ }^{6,7}$ A variety of supranuclear techniques have been described, which may be appropriate to teach to resident surgeons. These include Davis and Lindstrom's tilt and tumble, Pandit and Oetting's pop and chop, and Starr's pop and prechop. ${ }^{3,8,9}$ In a retrospective study of cataract surgery performed by trainees, Starr reported significantly lower levels of energy utilization, resulting in better corneal clarity scores using his pop and prechop technique. ${ }^{8}$ Similarly, the present study found that significant reductions in phaco energy could be achieved by novice resident surgeons performing pop and chop in their first 60 cases. In this study, pop and chop cases were all supervised by a single attending surgeon because he has greater familiarity with this technique and greater experience in teaching it to novice surgeons. A previous publication reported low and zero CDE phaco in resident cases using the pop and chop technique performed under the supervision of that and other attending surgeons. ${ }^{10}$ In several studies, it is found that lower energy has been associated with improved outcomes. ${ }^{6-8,11}$ Further studies are required to show whether the lower energy levels that we observed while using pop and chop will produce improved surgical outcomes in resident cataract surgery. 
The results from this study also clearly demonstrate that pop and chop is significantly faster than divide and conquer when performed by resident surgeons. Shorter case times have been reported to be associated with less endothelial cell loss and fewer surgical complications. ${ }^{6,11}$ On average, it was found that case time was 27.8 minutes ( $95 \% \mathrm{CI}=24.9-31.0$ ) for the divide and conquer method and 15.0 minutes $(95 \%$ $\mathrm{CI}=13.4-16.7)$ for the pop and chop method. In a previous study on resident cataract surgery, Hosler et al reported that resident case times improved from an average of 63 minutes at the beginning of the academic year to 27 minutes at the end of the academic year. ${ }^{12}$ In the present study, although surgical cases were observed early in training, the average case times were clearly reduced by the exclusion of complicated and difficult cases. However, based on the data from both the studies, a case time of $\sim 30$ minutes could be used as a reasonable goal for resident surgeons for performing uncomplicated cases of divide and conquer. In addition, goals for shorter case times could be reasonable when using supranuclear chopping techniques. From the present study, it was found that the shorter case times that were observed with pop and chop suggest that novice residents can quickly master this technique with measurable advantages in surgical efficiency in their first 60 cases.

In this study, the overall rate of intraoperative complications was $5.6 \%$ with divide and conquer and $7.5 \%$ with pop and chop in the first 60 cases performed by two resident surgeons. These values are consistent with the results of previous studies that report rates of vitreous loss in resident cases ranging from $6 \%$ to $15 \% \cdot{ }^{13,14}$ Randleman et al reported that the complication rate in resident cases clearly decreases after the first 80 cases and continues to decline as residents gain more experience. ${ }^{2}$ Although the present study observed cases early in residency training, based on the data obtained, it was concluded that pop and chop seems to be as safe as divide and conquer for novice surgeons. In a study on more experienced trainees performing pop and prechop, Christopher Starr found a significantly lower rate of vitreous loss $(0.9 \%)$ and concluded that supranuclear chopping is safer than endocapsular cracking or chopping for novice surgeons. ${ }^{8}$ Further studies comparing these nucleofractis techniques over the course of an entire residency year would be beneficial to confirm this hypothesis.

\section{Conclusion}

The present study demonstrated that pop and chop is safe to be taught to novice resident surgeons as an initial method of nuclear disassembly. This technique is faster and uses less energy than divide and conquer in early residency training. In addition, the data from this study suggest that the learning curve for this procedure is short, which allows residents to achieve proficiency in performing this quickly. Further studies are required to better define the role of this technique in residency education.

\section{Acknowledgments}

The authors would like to acknowledge Sarah C DeShields for her assistance in statistical analysis and preparation of figures and tables for this paper. The authors would also like to thank Kyle Den Beste for his assistance in reviewing charts and gathering data for this study. This material is the result of work supported with resources and the use of facilities at the Hampton VA Medical Center, Hampton, VA. The contents of this study do not represent the views of the Department of Veterans Affairs or the United States Government.

\section{Disclosure}

The authors report no conflicts of interest in this work.

\section{References}

1. Gimbel, HV. Divide and conquer nucleofractis phacoemulsification (development and variations). J Cataract Refract Surg. 1991;17: 281-291.

2. Randleman J, Wolfe JD, Woodward M, Lynn MJ, Cherwek D, Srivastava SK. The resident surgeon phacoemulsification learning curve. Arch Ophthalmol. 2007;125(9):1215-1219.

3. Pandit RT, Oetting TA. Pop-and-chop nucleofractis. J Cataract Refract Surg. 2003;29:2054-2056.

4. Chang DF. Learning Phaco Chop: Pearls and Pitfalls. New Orleans, LA: American Society of Cataract and Refractive Surgery; 2016.

5. Parlitsis G, Hussnain S, St Clair R, Lai E. Resident phacoemulsification using two distinct surgical techniques. Invest Ophthal Vis Sci. 2012; 53:6734.

6. DeBry P, Olson RJ, Crandall AS. Comparison of energy required for phaco-chop and divide and conquer phacoemulsification. J Cataract Refract Surg. 1998;24:689-692.

7. Wong T, Hingorani M, Lee V. Phacoemulsification time and power requirements in phaco chop and divide and conquer nucleofractis techniques. J Cataract Refract Surg. 2000;26:1374-1378.

8. Starr CE. Pop and prechop: a safer cataract technique for the beginning surgeon. Cataract Refract Surg Today. 2011;59-62.

9. Davis EA, Lindstrom RL. Tilt and tumble phacoemulsification. Dev Ophthalmol. 2002;34:44-58.

10. Zero energy phaco possible without a laser [webpage on the Internet]. Healio, Ocular Surgery News; 2015 [cited November 10, 2015]. Available from: http://www.healio.com/ophthalmology/cataract-surgery/ news/print/ocular-surgery-news/\%7Bb804afff-70a6-48c9-b08c37d149d60155\%7D/zero-energy-phaco-possible-without-a-laser. Accessed August 18, 2016.

11. Pirazzoli B, D’Eliseo D, Ziosi M, Acciarri R. Effects of phacoemulsification time on the corneal endothelium using phacofracture and phaco chop techniques. J Cataract Refract Surg. 1996;22(7):967-969.

12. Hosler RH, Scott IU, Kunselman AR, Wolford KR, Oltra EZ, Murray WB. Impact of resident participation in cataract surgery on operative time and cost. Ophthalmology. 2012;119:95-98.

13. Oetting TA. Surgical competency in residents. Curr Opin Ophthalmol. 2009;20(1):56-60.

14. Arbisser LB, Charles S, Howcroft M, Werner L. Management of vitreous loss and dropped nucleus during cataract surgery. Ophthalmol Clin North Am. 2006;19(4):495-506. 
Clinical Ophthalmology

\section{Publish your work in this journal}

Clinical Ophthalmology is an international, peer-reviewed journal covering all subspecialties within ophthalmology. Key topics include: Optometry; Visual science; Pharmacology and drug therapy in eye diseases; Basic Sciences; Primary and Secondary eye care; Patien Safety and Quality of Care Improvements. This journal is indexed on

PubMed Central and CAS, and is the official journal of The Society of Clinical Ophthalmology (SCO). The manuscript management system is completely online and includes a very quick and fair peer-review system, which is all easy to use. Visit http://www.dovepress.com/ testimonials.php to read real quotes from published authors. 